\title{
Effect of Psidium guajava L on Biofilm Forming Multidrug Resistant Extended Spectrum Beta Lactamase (ESBL) Producing Pseudomonas aeruginosa
}

\author{
Bhagawati Khadka ${ }^{1}$, Moni Mahato ${ }^{2}$, Reshma Tuladhar ${ }^{1 *}$, Anjana Singh ${ }^{1}$ \\ ${ }^{1}$ Central Department of Microbiology, Tribhuvan University, Kirtipur, Kathmandu, Nepal \\ ${ }^{2}$ KIST Medical College and Teaching Hospital, Lalitpur, Nepal
}

"Corresponding author: Dr. Reshma Tuladhar, Central Department of Microbiology, Tribhuvan University, Kathmandu, Nepal; Email: reshma.tuladhar@microbiotu.edu.np

\begin{abstract}
Objectives: In order to investigate the relative efficacy of Psidium guajava L (guava) leave, in vitro antibacterial effect of ethanolic extract and leaf tea of guava against pathogenic Pseudomonas aeruginosa was carried out.
\end{abstract}

Methods: This study was carried out at microbiology laboratory of KIST medical college and teaching hospital, Lalitpur. Pseudomonas aeruginosa was isolated from different clinical samples. Antibiotic susceptibility of the isolates was performed by Kirby-Bauer disk diffusion method. Biofilm formation was detected by microtitre culture plate method and ESBL production by combine disk methods. Antimicrobial activity of guava leaf tea and ethanol extract of leaf were determined by agar well diffusion method.

Results: Ethanol extract of fresh guava leaves exhibited higher antibacterial activity than dry and fresh leaves tea, but significantly less than the standard antibiotics. In this study, $7 \%$ of the total bacterial isolates were P. aeruginosa with $65.30 \%$ of these isolates being MDR. Similary, significant number of these MDR strains ie. $83.67 \%$ of these P. aeruginosa isolates produced biofilm. While as $6.12 \%$ of the isolates were ESBL producer.

Conclusion: The leaves extract of guava have shown effective result against $P$. aeruginos $a$ and could serve as good source of antibacterial agents. Guava leaves extract can be an economic alternative to antibiotics. However, active compound of this extract need to be purified and pharmacologically tested before its application.

Key words: Guava, Pseudomonas aeruginosa, antibacterial activity, AST

\section{INTRODUCTION}

Infectious diseases are the major cause of death in the developing countries. Although the global burden of infectious disease was reduced by using antimicrobial agents the spread of resistant pathogen has diminished the effectiveness of the antibiotics (Bisht et al. 2016). An inheritent resistant bacteria, Pseudomonas aeruginosa, belonging to Gram-negative aerobic bacilli of Pseudomonadaceae family, are posing challenge in treatment of various infections it cause such as: urinary tract infections, respiratory infections, otitis media, skin and soft tissue infections, bone and joint infections, and bacteremia. Besides, it can also cause serious systemic infections particularly in people with compromised immune systems including patients of

Date of Submission: October 31, 2019

Published Online: December, 2019 burn suffer, cystic fibrosis, cancer and AIDS (Neopane et al. 2017). It has emerged as one of the leading causes of nosocomial infections. P. aeruginosa is the sixth most frequently isolated nosocomial pathogen, causing $7.3 \%$ of all hospital acquired infection in the US (Weiner et al. 2016). Infections due to P. aeruginosa are difficult to eradicate due to their intrinsic resistance as well as their ability to acquire resistance to different antibiotics. The resistance is due to over expression of efflux pump, acquisition of extended spectrum $\beta$-lactamases (ESBLs) and metallo- $\beta$-lactamases (MBLs), target site or outer membrane modification, porin mutations and plasmid enzymatic modification (Heydari and Eftekhar 2015). P. aeruginosa can form biofilms, which exponentially increase antibiotic

Date of Acceptance: November 30, 2019

DOI: https:/ / doi.org/10.3126/tujm.v6i0.26574 
resistance. The three exo-polysaccharides that mainly contribute to the biofilm formation in this bacteria are; alginate, Psl (Polysaccharides Synthesis Locus), and Pel (Pellicle). Alginate confers additional protection against antimicrobials and the immune system while Psl and Pel contribute to aggregation and adherence (Nithyalakshmi et al. 2015). Hence, dealing with multidrug resistant strain of these bacteria is challenging.

Opting for an alternative natural plant product to inhibit this bacteria we have selected Guava leave (Psidium gaujava L). Guava leave (Psidium gaujava L), a phyto-therapeutic plant used in folk medicine, and is believed to have active antimicrobial compound which can be used to treat and cure various diseases (Mailoa et al. 2014). Different parts of the plant have been used in traditional medicine against ailments like malaria, gastroenteritis, vomiting, diarrhoea, dysentery, wounds, ulcers, toothache, coughs, sore throat, inflamed gums, controlling of diabetes, hypertension, and obesity (Biswas et al. 2013).

Study in Nepal has shown the association of nosocomial infections with multidrug resistant (MDR) P. aeruginosa. The occurrence of biofilm and MDR bacteria in a hospital setting possess a therapeutic problem, as well as a serious concern for infection control management. Therefore, minimizing the use of antibiotics and possibly substituting with antimicrobial compound from natural source can be an alternative to subside the growing antibiotic resistant problem. This study was therefore undertaken to evaluate the antimicrobial activity of guava leaf tea and extract on $P$. aeruginosa isolated from various clinical samples.

\section{MATERIALS AND METHODS}

$P$. aeruginosa was isolated from different clinical samples such as pus/wound, blood, sputum and urine at KIST medical college and teaching hospital, Lalitpur, a tertiary care hospital from $15^{\text {th }}$ June 2017 to $15^{\text {th }}$ Dec 2017. They were identified by standard microbiological techniques. Antibiotic susceptibility of the isolates was performed by Kirby-Bauer disk diffusion method on Mueller Hinton agar as per CLSI guidelines (CLSI 2015). The isolates resistant to $\geq 1$ agent in $\geq 3$ antimicrobial categories were considered multidrug resistant (MDR). ESBL production was screened using two disks, ceftazidime $(30 \mu \mathrm{g})$ and cefotaxime $(30 \mu \mathrm{g})$ according to the CLSI guidelines. An inhibition zone of $\leq 22 \mathrm{~mm}$ for ceftazidime and $\leq 27 \mathrm{~mm}$ for cefotaxime indicated a probable ESBL producing strain which was further confirmed by combination disc method.

\section{Detection of biofilm production}

Biofilm production was detected by microtitre culture plate method (TCP), a quantitative test as described by Christensen et al. (1995). Organisms isolated from fresh agar plates were inoculated in $10 \mathrm{~mL}$ of trypticase soy broth with $1 \%$ glucose. Broths were incubated at $37^{\circ} \mathrm{C}$ for $24 \mathrm{~h}$. The cultures were then diluted 1:100 with fresh medium. Individual wells of sterile 96 well flat bottom polystyrene tissue culture treated plates were filled with $200 \mu \mathrm{L}$ of the diluted cultures. The control organisms used was P. aeruginosa ATCC 27853. Negative control wells contained inoculated sterile broth without inoculum. The plates were incubated at $37^{\circ} \mathrm{C}$ for $24 \mathrm{hr}$. After incubation, contents of each well were removed by gentle tapping. The wells were washed with $0.2 \mathrm{~mL}$ of phosphate buffer saline ( $\mathrm{pH}$ 7.2) four times. This removed free floating bacteria. Biofilm formed by bacteria adherent to the wells were fixed by $2 \%$ sodium acetate and stained by crystal violet $(0.1 \%)$. Excess stain was removed by using deionized water and plates were kept for drying. Optical density (OD) of stained adherent biofilm was obtained by using micro ELISA auto reader at wavelength $570 \mathrm{~nm}$. The experiment was performed in triplicate and repeated three times. Interpretation of biofilm production was done according to the criteria of Stepanovic et al. (2000).

\section{Preparation of guava tea and ethanol extract}

Guava leaf samples were randomly collected from guava tree in Kathmandu Valley. The leaves were washed with sterile distilled water. Extract first (fresh leaf tea) was prepared by boiling ten leaves in conical flask containing $200 \mathrm{ml}$ of distilled water. Extract second (dry leaf tea) was prepared by boiling freeze dried leaves of the same weight and numbers. Extract third and fourth (ethanol extract of fresh and dried leaves respectively) were prepared by using absolute ethanol as a solvent. The leaves pieces were added to solvent in sterile flask and wrapped in aluminum foil to avoid evaporation and exposed to light. The flasks were placed on a platform shaker at $70 \mathrm{rpm}$ for 3 days. The mixtures were then transferred to tubes and centrifuged for $10 \mathrm{~min}$ at $4,000 \mathrm{rpm}$. The supernatant was collected and stored at $4^{\circ} \mathrm{C}$ until use. 
Determination of antimicrobial activity of guava leave tea against $P$. aeruginosa

Antimicrobial activity of tea and extract were done by agar diffusion method in triplicate on Mueller Hinton Agar (MHA) plates according to the guidelines of the National Committee for Clinical Laboratory Standards. Prior to streaking the plates with bacteria, $5 \mathrm{~mm}$ diameter wells were punched into the medium using a sterile borer. The surface of the agar plate was swabbed with 0.5 McFarland standarized broth culture of isolated P. aeruginosa and allowed to dry for $5 \mathrm{~min}$. Each $100 \mu \mathrm{l}$ aliquots of extract and leaves tea were dispensed into each well of MHA plates swabbed with bacteria. For positive control tobramicin was placed at the centre of the plate. Ethanol was used as negative control. $P$. aeruginosa ATCC 27853 was used as positive control organism. The plates were incubated for 24 hours and zone of inhibition was noted.

\section{RESULTS}

Out of total 3000 specimen processed, bacterial growth was observed in $32 \%$ samples and $P$. aeruginosa was isolated from $7 \%$ of the total sample. Bacterial isolates other than P. aeruginosa accounted for $25 \%$ of the total (Figure 1).
Antibiotic susceptibility pattern and prevalence of MDR P. aeruginosa

Most of the isolates were sensitive towards colistin and resistant towards cefepime followed by imipenem and cefotaxime (Table 1). Among the total P. aeruginosa isolated in this study $65.3 \%(n=32)$ were MDR (Figure 2).

\section{Biofilm production detection in P. aeruginosa}

Out of 49 isolates of $P$. aeruginosa $35(71.40 \%)$ were strong biofilm producer, while 6 (12.24\%) were weak producers and $8(16.32 \%)$ were non-biofilm producers (Figure 3).

\section{ESBL production in $P$. aeruginosa}

Among the total P. aeruginosa isolates, 3 (6.12\%) were found to be ESBL producer. Significant numbers of biofilm producers were MDR isolates $(p<0.05)$ (Table 2).

\section{Antimicrobial activity of guava leave}

Guava leaf tea (fresh and dry leaves tea) showed antimicrobial activity against $P$. aeruginosa isolates. However, compared to the antimicrobial activity of standard antibiotic tobramicin, the zone of inhibition shown by guava leaves was less (Figure 4). The inhibition zone given by fresh leaves for both guava leaf tea and ethanol extract were higher compared to the dry leaves (Figures 4 and 5).

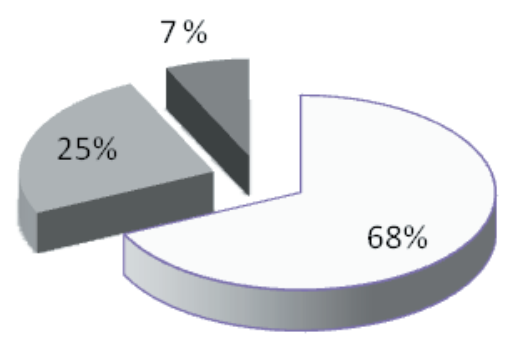

\author{
No Growth \\ Total Bacteria \\ other than P. \\ aeruginosa \\ - P. aeruginosa
}

Figure 1: Growth pattern of bacterial isolates in clinical samples

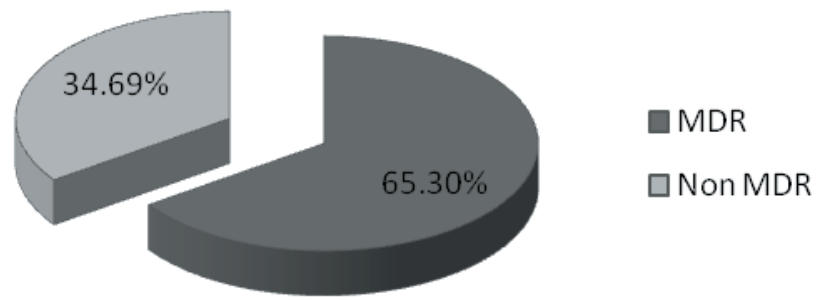

Figure 2: Percentage of MDR strain from the total isolates of $P$. aeruginosa 


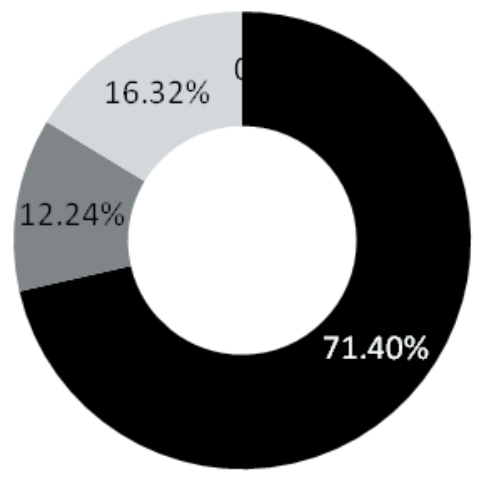

- Strong biofilm producer

Weak biofilm producer

Non-biofilm producer

Figure 3: Percentage of biofilm producing $P$. aeruginosa

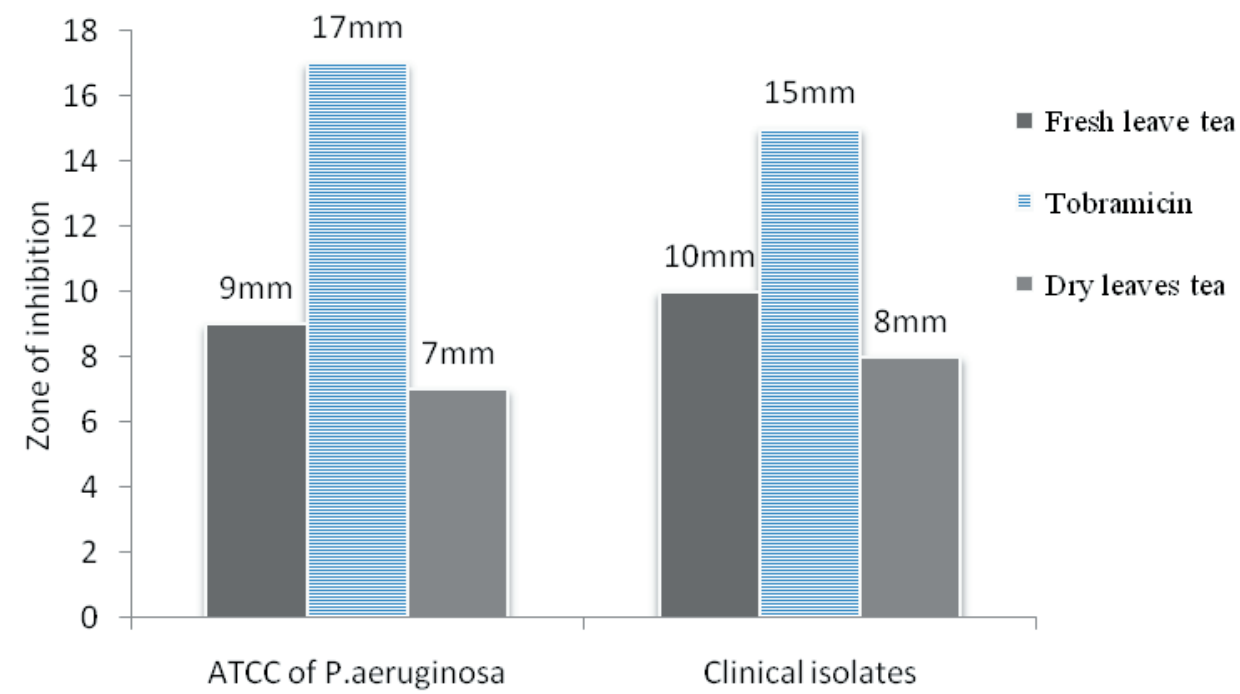

Figure 4: Antimicrobial activity of guava leaves tea and tobramicin against $P$. aeruginosa

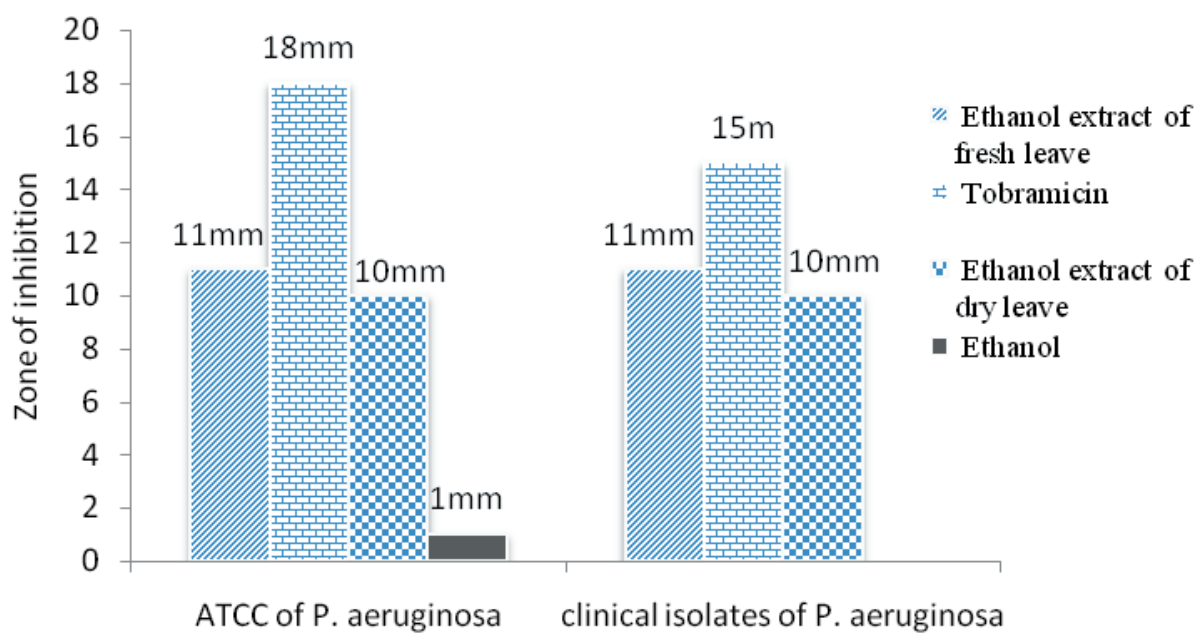

Figure 5: Antimicrobial activity of ethanol extract of guava leaves and tobramicin against P. aeruginosa 
Table 1: Antibiotic susceptibility pattern of $P$. aeruginosa

\begin{tabular}{lccc}
\hline Antibiotic & Sensitive $\%$ & Intermediate $\%$ & Resistant \% \\
\hline Piperacillin & $31(63.3)$ & - & $18(36.7)$ \\
Piperacillin/Tazobactam & $27(55.1)$ & $3(6.1)$ & $19(38.8)$ \\
Ceftriaxone & $20(40.8)$ & $7(14.3)$ & $22(46.9)$ \\
Ceftazidime & $22(44.9)$ & $4(8.2)$ & $23(46.9)$ \\
Cefepime & $16(32.7)$ & - & $33(67.3)$ \\
Aztreonam & $29(59.2)$ & $6(12.2)$ & $14(28.6)$ \\
Gentamicin & $29(59.2)$ & - & $20(40.8)$ \\
Amikacin & $24(49.0)$ & $1(2.0)$ & $24(49.0)$ \\
Imipenem & $19(38.8)$ & $1(2.0)$ & $29(59.2)$ \\
Meropenem & $24(49.0)$ & $2(4.1)$ & $23(46.9)$ \\
Ciprofloxacin & $30(61.0)$ & - & $19(38.8)$ \\
Colistin & $46(93.9)$ & - & $3(6.1)$ \\
Polymyxin-B & $32(65.3)$ & $5(10.2)$ & $12(24.5)$ \\
Cefotaxime & $20(40.8)$ & $3(6.1)$ & $26(53.1)$ \\
Tobramycin & $29(59.2)$ & $1(2.0)$ & $19(38.8)$ \\
\hline
\end{tabular}

Table 2: Comparison between MDR and biofilm production

\begin{tabular}{|c|c|c|c|c|}
\hline \multirow{2}{*}{ MDR } & \multicolumn{2}{|c|}{ Biofilm producer } & \multirow{2}{*}{ Total (\%) } & \multirow{2}{*}{$\mathrm{p}$-value } \\
\hline & Positive (\%) & Negative (\%) & & \\
\hline Positive & $31(96.8)$ & $1(3.2)$ & $32(100.0)$ & 0.02 \\
\hline Negative & $10(58.82)$ & $7(41.18)$ & $17(100.0)$ & \\
\hline Total & 41 (83.67) & $8(16.33)$ & $49(100.0)$ & \\
\hline
\end{tabular}

\section{DISCUSSION}

$P$. aeruginosa is an opportunistic nosocomial pathogen of great important due to its resistance to multiple antibiotics (Goel et al. 2013). In this study, prevalence of $P$. aeruginosa from different clinical samples was found to be $27.22 \%$ which is less compared to Ali et al. (2015) and Goel et al. (2013) who reported that 39\% and $37.7 \%$ of $P$. aeruginosa were isolated from ICU patients in Pakistan and India, respectively.

High prevalence of MDR P. aeruginosa (65.3\%) was reported in this study which is similar to the finding of Fatima et al. (2012) where MDR isolates accounted for $73.9 \%$. This finding is also corroborated with MDR P. aeruginosa in Southeast Asia, where $71 \%$ reported during 2007-2009 (Suwantarat and Carroll 2016). A high prevalence of MDR $P$. aeruginosa seen in hospital acquired infections was due to selective pressure exerted by over usage of broad spectrum antibiotics. However, the emergence of MDR is related to the empirical use of antibiotics rather than the rational use of broad-spectrum antibiotics before the sample collection.

Prevalence of ESBL producing P. aeruginosa was 6.12\%. Among the ESBL producer $66.67 \%$ were MDR and $6.25 \%$ MDR were ESBL producer, which is similar to a study by Stepanovic et al. (2000). Shaikh et al. (2015) reported $25.13 \%$ isolates of $P$. aeruginosa were ESBL positives. The ESBL producing $P$. aeruginosa isolates exhibited co-resistance against most of the antibiotics tested.

In this study, $83.67 \%$ isolates of $P$. aeruginosa were biofilm producer. Maita and Boonbumrung (2014) reported $79.4 \%$ biofilm producer in Thailand. Similar to this study $(89.3 \%)$ was reported by Sharma and Chaudhary (2015) but lower (48.8\%) by Tamaraiselvi et al. (2015). The biofilm production was independent of antibiotic susceptibility profile of the bacteria. When the degree of adhesion of the biofilm is high, the penetration of the antimicrobial compound into its structure is reduced resulting in the increased resistance of the bacteria.

Antibacterial activity of ethanol extract of fresh leaves showed higher activity $(11 \mathrm{~mm})$ followed by ethanol extract of dry leaves and least activity by dry guava tea solution $(7 \mathrm{~mm})$. Guava leaf extract and leaf tea exhibited some antibacterial activity against $P$. aeruginosa but less compared to the standard antibiotic tobramycin. Biswas et al. (2013) reported that $P$. guajava has antibacterial effect against both Gramnegative and Gram-positive bacteria. It was due to the presence of alkaloids, flavonoids, tannins, saponins, 
glycosides and terpenoids in the leaves extracts of $P$. guajava (Savoia 2012). These phytochemicals have in vitro inhibitory activity against some clinical bacterial isolates. In Brazil, Sanches et al. (2005) reported that the aqueous extracts of $P$. guajava leaves, roots and stem bark were active against the Gram positive bacteria but not against Gram negative species. This can be due to the outer membrane of Gram negative bacteria which act as barrier for penetration of numerous antibiotic molecules. Besides, the enzymes present in the periplasmic space have ability to break down foreign molecules.

\section{CONCLUSION}

$P$. aeruginosa is a pathogen of interest in most of the hospital acquired infection. Increase in the drug resistant $P$. aeruginosa is a great challenge in treatment of infections caused by it. Since crude ethanol extract of guava leave and its tea was able to inhibit $P$. aeruginosa, the compound present in guava leaves extract and tea contain some antimicrobial compounds which can effectively control pathogenic bacteria. It has potential for use in therapy against infections caused by pathogens. Thus we recommend additional test from pure extracts along with further pharmacological evaluation.

\section{ACKNOWLEDGMENTS}

Authors are thankful to UGC (University Grants Commission), Nepal for providing financial support.

\section{CONFLICT OF INTEREST}

The authors declare no conflict of interest.

\section{REFERENCES}

Ali Z, Mumtaz N, Naz SA, Jabeen N and Shafique M (2015). Multi-drug resistant Pseudomonas aeruginosa. A threat of nosocomial infections in tertiary care hospitals. J Pak Med 65: 10-21.

Bisht R, Chanyal S and Agrawal PK (2016). Antimicrobial and phytochemical analysis of leaf extract of medicinal plants. Asian J Pharm Clinic Res 9: 131-136.

Biswas B, Rogers K, McLaughlin F, Daniels D and Yadav A (2013). Antimicrobial activities of leaf extracts of guava (Psidium guajava L.) on two Gram-negative and Gram-positive bacteria. Int $J$ of Microbiol 7: 122-129.

Centers for Disease Control and Prevention (2015). Health care associated Infections. CDC, Atlanta,
USA 4: 1-14.

Christensen GD, Simpson WA and Younger JA (1995). Adherence of coagulase negative Staphylococci to plastic tissue cultures: a quantitative model for the adherence of Staphylococci to medical devices. J Clin Microbiol 22: 996-1006.

Fatima A, Naqvi SB, Kkali SA, Perveen S and Jabeen $S$ (2012). Antimicrobial susceptibility pattern of clinical isolates of Pseudomonas aeruginosa isolated from patients of lower respiratory tract infections. Springer Plus 1: 70.

Goel V, Sumati A, Hogade SA and SG Karadesai (2013). Prevalence of extended spectrum $\beta$-latamases, AmpC beta lactamase, and metallo- $\beta$ lactamase producing Pseudomonas aeruginosa and Acinetobacter baumanni in an intensive care unit in a tertiary care hospital. J Sci Sco 40: 28-31.

Heydari S and Eftekhar F (2015). Biofilm formation and $\beta$-Lactamase production in burn isolates of Pseudomonas aeruginosa. Jundishapur J Microbio 4: 111-123.

Mailoa MN, Mahendradatta M, Laga M and Djide $\mathrm{N}$ (2014). Antimicrobial activities of tannins extract from guava leaves (Psidium gaujava $\mathrm{L}$ ) on pathogens microbial. Int J of Sci and Tech Res 1: 236-241.

Maita P and Boonbumrung K (2014). Association between biofilm formation of Pseudomonas aeruginosa clinical isolates versus antibiotics resistant and genes involved with biofilm. J Chem and Pharma Res 6: 1022-1028.

Neopane P, Nepal HP, Gautam R, Paudel R, Ansari $S$ and Shrestha $S$ (2017). Is there correlation of biofilm formation with multidrug resistance and esbl production in Pseudomonas aeruginosa. European J of Biomed 4 : 366-372.

Nithyalakshmi J, Akila K, Mohanakrishnan K and Sumathi G (2015). Evaluation of resistance profile of Pseudomonas aeruginosa with reference to biofilm production - An emerging challenge. J of Den and Med Sci 14: 13-18.

Sanches RN, Garcia AD, Schiavini SM, Nakamura CV and Filho DB (2005). An evaluation of antibacterial activities of $P$. guajava (L). An Int J 48: 429-436.

Savoia D (2012). Plant-derived antimicrobial 
compounds. Future Microbio 7: 979-90.

Shaikh S, Fatima J, Shakil S, Mohd S, Rizyi D and Amijakamal M (2015). Prevalence of multidrug resistant and extended spectrum beta-lactamase producing Pseudomonas aeruginosa in a tertiary care hospital. Saudi J Bio Sci 22: 62-64.

Sharma I and Chaudhary D (2015). Detection of Pel A gene in $P$. aeruginosa from clinical samples using polymerase chain reaction with reference to biofilm production in N E India. Ind J Res 4: 119-121.

Stepanovic S, Vukovi D and Hola V (2000). Quantification of biofilm in microtiter plates: overview of testing conditions and practical recommendations for assessment of biofilm production by Staphylococci. APMIS 115: 891-9.
Suwantarat N and Carroll KC (2016). Epidemiology and molecular characterization of multi-drug resistant Gram negative bacteria in Southeast Asia. Antimicrob Res and Infect Cont 5: 1-8.

Tamaraiselvi S, Swarma SR, Madhavan R and Gomathi S (2015). Biofilm production and multi drug resistant among the isolates from acute and chronic wound infection. Int J Pharma Bio Sci 6: 1197-1203.

Weiner LM, Webb AK, Limbago B, Dudeck MA, Patel J, Kallen AJ, Edwards JR and Sievert DM (2016). Antimicrobial resistant pathogens associated with healthcare-associated infections: summary of data reported to the national healthcare safety network at the Centres for Disease Control and Prevention, 20112014. Infect Cont and Hosp Epid 4: 1-14. 